\title{
Be Star Surveys in Open Clusters with Balmer-line Photometry
}

\author{
Gemma Capilla and Juan Fabregat \\ Departamento de Astronomía, Universidad de Valencia, 46100 \\ Burjassot, Spain \\ Deborah Baines \\ Department of Physics \& Astronomy, University of Leeds, U.K.
}

\begin{abstract}
We present CCD $\mathrm{H} \alpha$ and $\mathrm{H} \beta$ photometry of young open clusters. We show that the comparison of the $\alpha$ and $\beta$ photometric indices provides an efficient tool for identifying emission line stars. We report on the discovery of several new Be stars.

The preliminary results of our survey are the following: i. the younger clusters (age $<10 \mathrm{Myr}$ ) are almost lacking of Be stars, ii. clusters in the age interval 10-30 Myr are rich in Be stars. Almost all of them are of spectral types earlier than B5, while late-type Be stars are scarce. These results point towards an evolutionary interpretation of the $\mathrm{Be}$ phenomenon, in the sense that Be stars are close to the end of their main sequence lifetime.
\end{abstract}

\section{Introduction}

The study of the abundances of Be stars in open clusters of different ages is a fundamental issue in the discussion on the evolutionary status of Be stars. This kind of studies is, however, strongly limited by the scarcity of emission line star surveys in open clusters present in the literature.

In this work we present the first results of a survey for emission line objects in young open clusters which we have recently undertaken. We are using the technique of CCD imaging photometry through interference filters centered in the $\mathrm{H} \alpha$ and $\mathrm{H} \beta$ Balmer lines.

\section{Observations and reduction procedure}

The observations were done during two runs in December 1995 and November 1998 at the Calar Alto Observatory (Almería, Spain). The first run was performed with the $1.23 \mathrm{~m}$ telescope of the Centro Astronómico Hispano Alemán, and the second one with the $1.52 \mathrm{~m}$. telescope of the Observatorio Astronómico Nacional.

In each telescope we used two pairs of interference filters centered on $\mathrm{H} \alpha$ and $\mathrm{H} \beta$ Balmer lines. Each pair consisted of a wide and a narrow filter. 


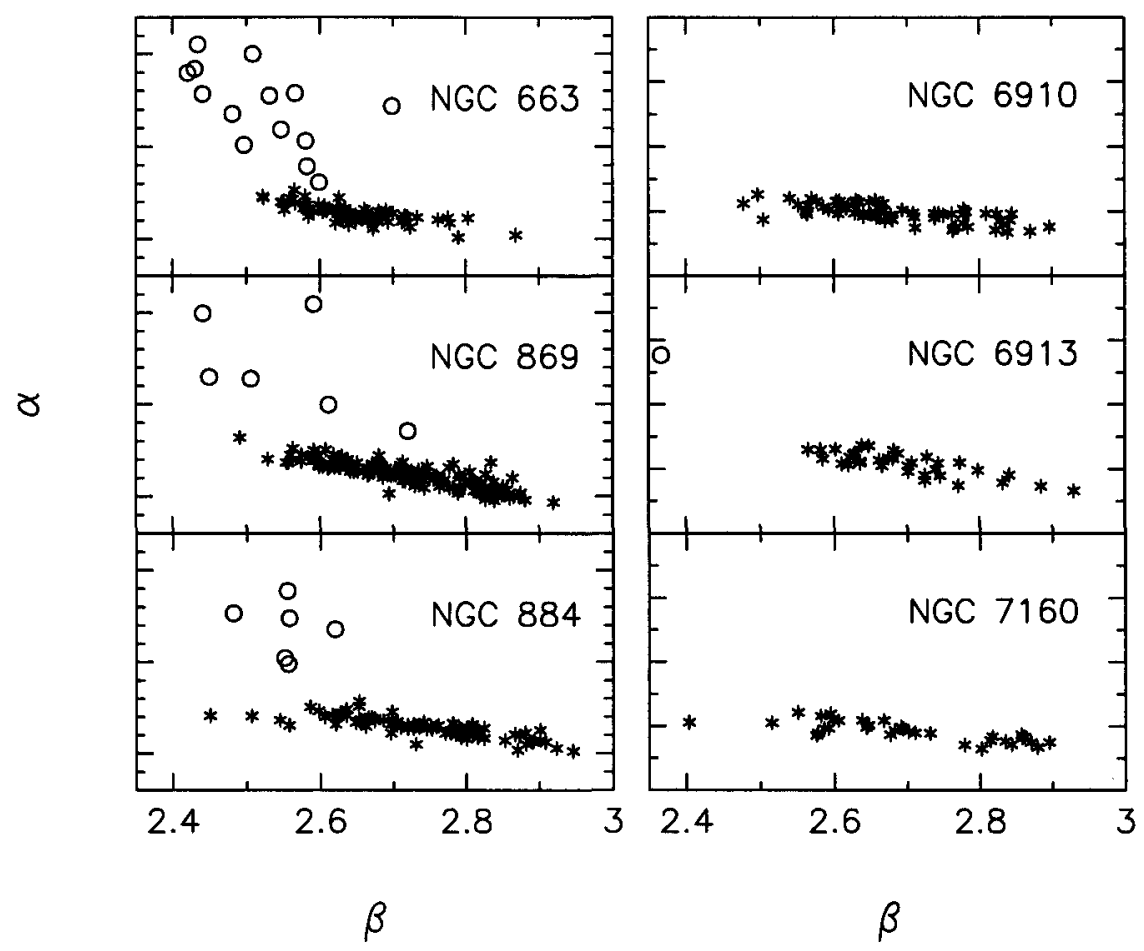

Figure 1. $\quad \alpha-\beta$ photometric diagram for the six observed clusters. Be stars are represented with open circles.

The data have been reduced using the standard procedures in IRAF. For every frame we have obtained the instrumental magnitudes of the stars. We have transformed the instrumental magnitudes into indices $\alpha$ and $\beta$ by substracting the magnitude in the wide filter from the magnitude in the narrow one.

We have transformed our instrumental $\beta$ indices into the standard Crawford \& Mander (1966) system. We have used as standards the stars in common with Crawford et al. $(1970,1977)$. As there is not a widely accepted $\mathrm{H} \alpha$ photometric system, we have worked with the instrumental indices obtained in our observations. Note that the instrumental $\alpha$ systems are different in the two runs, which explain the different scales in the figure.

There is a good correlation between $\mathrm{H} \alpha$ and $\mathrm{H} \beta$ for B stars, so when plotting the two indices for objects without emission a well-defined sequence is apparent. Since $\mathrm{H} \alpha$ emission is much stronger than $\mathrm{H} \beta$ emission for $\mathrm{B}$ emission-line stars, such objects appear significantly above the main sequence. In Figure 1 we have represented the photometric $\alpha-\beta$ plane for the six observed open clusters. Be stars have been represented with a different symbol. 
The presented photometry is complete to magnitude 15 . At the reddening and distance of all observed clusters this means that our survey is complete through all the B-type main sequence, except for NGC 663 in which we reach the spectral type B8-9V.

\section{Analysis of individual clusters}

\subsection{NGC 663}

NGC 663 is the galactic cluster with the highest frequency of Be stars. In our survey we have found 14 Be stars. Three among them (stars 61, 102 and 175) are previously undetected emission-line objects. From the position of Be stars in the photometric diagrams we can deduce that all of them are early-type Be stars, i.e., earlier than B5, with only one exception, namely star 175 . The magnitude of this star, at the cluster distance and reddening, is compatible with a spectral type B6V.

\subsection{NGC 869 (h Persei)}

We have detected 6 Be stars, among which two are new detections (stars 1058 and 1278). Only one star appears to be later than B5, namely star 1058. Its magnitude assuming the cluster distance and reddening would indicate a late A-type main sequence star, and so if this object is indeed a Be star it is a background object.

\section{3. $\quad$ NGC 884 ( $\chi$ Persei)}

We have detected 6 Be stars, all of them previously catalogued. All stars are of spectral types earlier than B5.

\subsection{NGC 6910}

No Be stars have been detected in this cluster.

\subsection{NGC 6913 (M 29)}

We have detected one previously known, early-type Be star, namely star 6 .

\subsection{NGC 7160}

No Be stars have been detected in this cluster. It should be noted that the brightest stars in this cluster are bright enough to saturate our CCD frames, so they are not included in the diagram. Among these stars is the well known Be star EM Cephei.

\section{Conclusions}

- The CCD Balmer-line photometry is an efficient tool to detect emission line stars in open clusters.

- Even in the most extensively studied clusters, like NGC 663 and h and $\chi$ Persei, every new survey always discovers new Be stars. This can be 
interpreted in the sense that for most stars the Be activity is present only during a small percentage of the time, and hence the currently assumed frequencies of Be stars could be underestimated.

- The younger clusters in our sample (NGC 6910, NGC 6913 and NGC 7160 ), with age $<10 \mathrm{Myr}$, are almost completely lacking of Be stars. Clusters in the age interval 14-25 Myr (NGC 663, NGC 869 and NGC 884) contain a large number of emission-line objects. This can be explained within the evolutionary hypothesis recently presented by Fabregat \& Torrejón (1999). They propose that the Be star phenomenon appears in the second half of the main sequence lifetime of a B star. B stars younger than $10 \mathrm{Myr}$ are still in the first half of the main sequence phase, and hence do not present the Be phenomenon.

- Almost all Be stars in the clusters within the age interval 14-25 Myr are of spectral types earlier than B5. This is also consistent with the evolutionary hypothesis. In this age interval, stars earlier than B5 are in the second half of the main sequence phase, while late-type stars are still in the first half.

Acknowledgments. We are grateful to the Observatorio Astronómico Nacional and the Centro Astronómico Hispano-Alemán for awarding telescope time for this project. IRAF is distributed by the National Optical Astronomy Observatories which are operated by the Association of Universities for Research in Astronomy, Inc., under contract to the National Science Foundation.

\section{References}

Crawford, D.L., Mander J. 1966, AJ 71, 144

Crawford, D.L., Glaspey, J.W., Perry C.L. 1970, AJ 75, 822

Crawford, D.L., Barnes, J.V., Hill G. 1977, AJ 82, 606

Fabregat, J., Torrejón, J.M. 1999 A\&A (in press) 